Ann. Zootech., I974, 23 (I), 89-IO4.

\title{
ENREGISTREMENT DU pH INTRAGASTRIQUE CHEZ LE PORC : VARIATIONS LIÉES A LA NATURE, A L'IMPORTANCE ET A L'INTERVALLE DES REPAS CHEZ L'ANIMAL EN FINITION
}

\author{
J.-P. LAPLACE \\ avec la collaboration technique de C. Germain \\ Laboratoire de Physiologie de la Nutrition, \\ Centre national de Recherches zootechniques, I. N. R. A., \\ 78350 Jouy en Josas \\ RÉSUMÉ
}

L'enregistrement continu du $\mathrm{pH}$ intragastrique in vivo a été effectué chez 6 porcs de race Large White pesant entre 60 et $68 \mathrm{~kg}$. L'électrode stomacale d'enregistrement est localisée à $2 \mathrm{~cm}$ du pylore après introduction par l'intermédiaire d'un tube gastrique permanent. L'électrode de référence est mise en place dans une canule cæcale. Quatre régimes ont été utilisés : 2 aliments standard, de croissance ou de finition, et 2 aliments de finition incorporant $4^{o}$ p. Ioo de lactosérum sec, doux ou acide. L'évolution des courbes de $\mathrm{pH}$, au cours de périodes d'une durée maximum de 8 heures, est décrite dans chacun des cas. Seul le premier repas de la journée induit une variation importante, dont l'amplitude et le décours permettent de caractériser un type de courbe particulier pour chaque aliment. L'importance ou le nombre des repas sont dépourvus d'influence marquante.

\section{INTRODUC'TION}

Parmi les facteurs de contrôle susceptibles d'intervenir dans la régulation de l'évacuation gastro-duodénale, l'acidité du chyme évacué par l'estomac ne peut être négligée. Le travail mathérnatique antérieurement effectué sur les résultats de l'étude quantitative de l'évacuation gastrique (LAPIACE et TOMASSONE, I970) nous avait amené à formuler un certain nombre d'hypothèses explicatives purement mécaniques en relation avec les effets du nombre et de la chronologie de distribution des repas, de leur volume ou de leur dilution. Par la suite, l'étude de la motricité gastro-duodé- 
nale (LAPLACE, I97 I et I972) réalisée en fonction de facteurs tels que taux de dilution et composition de l'aliment avait permis de constater la relative lenteur de l'évacuation gastrique d'un aliment comportant du maïs par rapport à un aliment à base d'orge. Cet effet permettait d'expliquer le caractère également lent de l'absorption des produits d'hydrolyse de l'amidon de maïs (RÍrRat et AUmaITRE, I97I) en dépit du fait qu'un tel amidon est digéré plus vite in vitro (DERRIEN, GaliaANT et AUMaITRE, I97I) et in vivo (FÉVRIER, RÉRAT et AUMATTRE, I97I) que des amidons de manioc ou de pomme de terre. Cette lenteur d'évacuation gastrique d'un repas à base de maîs avait été rattachée à l'interaction évidente des activités motrices respectives de l'antre gastrique et du duodénum. Cependant, la rapidité de l'évacuation gastrique est également déterminée par l'influence des propriétés physiques et chimiques du chyme porté au contact de la muqueuse réceptrice sensible du bulbe duodénal et du duodénum dans son ensemble. Dans ce sens, le $\mathrm{pH}$ du chyme évacué par l'estomac, comme la pression osmotique peuvent constituer des facteurs de régulation particulièrement agissants.

Pour cette raison, l'évolution du $\mathrm{pH}$ intragastrique, enregistré immédiatenent en avant du pylore, a été étudiée en continu en fonction des principaux facteurs précédemment envisagés : volume de la ration, nombre de repas, composition de 1'aliment (orge ou maiis). A titre comparatif, les effets d'aliments comportant 1'adjonction de lactosérums ont été envisagés.

\section{MATÉRIEL ET MÉTHODES}

Six porcs de race Large White, mâles castrés ou femelles, pesant entre 60 et $68 \mathrm{~kg}$ au début de leur utilisation expérimentale, ont été préparés chirurgicalement sous anesthésie générale : - Un tube en silicone médical (diamètre intérieur $6,35 \mathrm{~mm}$, diamètre extérieur 9,53 mm) est mis en place au niveau de l'antre gastrique selon la technique de Witzel (Markowitz, ARCHIBALD et DownIE, 1964) de façon à ce que son extrémité " digestive "soit ouverte à 2 ou $3 \mathrm{~cm}$ du pylore (fig. I). L'extrémité libre est extérioriséc à mi-longueur du cercle de l'hypocondre droit. - Une canule en silicone médical (diamètre intérieur I $2,7 \mathrm{~mm}$, diamètre cxtérieur $18,0 \mathrm{~mm}$ ) est placée dans l'extrémité aveugle du cæcum, et extériorisée au centre du creux du flanc droit.

La technique d'anesthésie, comme l'installation des animaux en cage, sont celles décrites antérieurement (LAPLACE et TOMASSONE, I970).

Pour les séances d'enregistrement, d'une durée maximum de 8 heures, on introduit dans le tube gastrique une électrode stomacale argent-chlorure d'argent d'un diamètre de $4 \mathrm{~mm}$ (BEсKMAN, réf. 39042). L'électrode de référence argent-chlorure d'argent à jonction de fibre (Beckman, réf. 40249), beaucoup plus volumineuse est mise en place dans la canule caccale. Ces électrodes sont reliées à un pH-mètre à lecture directe (BECKMAN, Zeromatic SS 3). Le signal est retransmis du pHmètre à un polygraphe enregistreur (type Dynograph (R) R. M. BECKMAN), par l'intermédiaire d'un adaptateur à résistance variable et d'un coupleur d'entrée $9806 \mathrm{~A}$. Ce procédé fournit donc la courbe ce l'évolution du $\mathrm{pH}$ en fonction du temps, tout en permettant la lecture directe à volonté. En ce qui concerne l'enregistrement continu sur polygraphe, les variations rapicles du signal sont éliminées au niveau de l'amplificateur de puissance qui agit alors comme filtre passe-bas. Le signal enregistré est ainsi une moyenne pondérée en fonction du temps du signal d'entrée. Ce processus permet de ne conserver que l'évolution effective du $\mathrm{pH}$ pré-pylorique, en effaçant les fluctuations brèves et de faible amplitude correspondant à l'effet immédiat des ondes contractiles qui parcourent l'antre. $\Lambda u$ demeurant, celles-ci peuvent être examinées conjointement par enregistrement continu du signal direct sur une piste parallèle du polygraphe.

Préalablement à chaque séance d'enregistrement, il est procédé à un étalonnage de I'enregistreur, à l'aide de solutions tampons de pH 4,or-6,86 ou 7,oo (Beckman, réf. 3005-30063007). Il est tenu compte de la température à laquelle travaillent les électrodes par ajustement au niveau du pH-mètre ( $20^{\circ}$ pour la solution tampon - $39^{\circ}$ dans l'animal). Un nouvel étalon- 


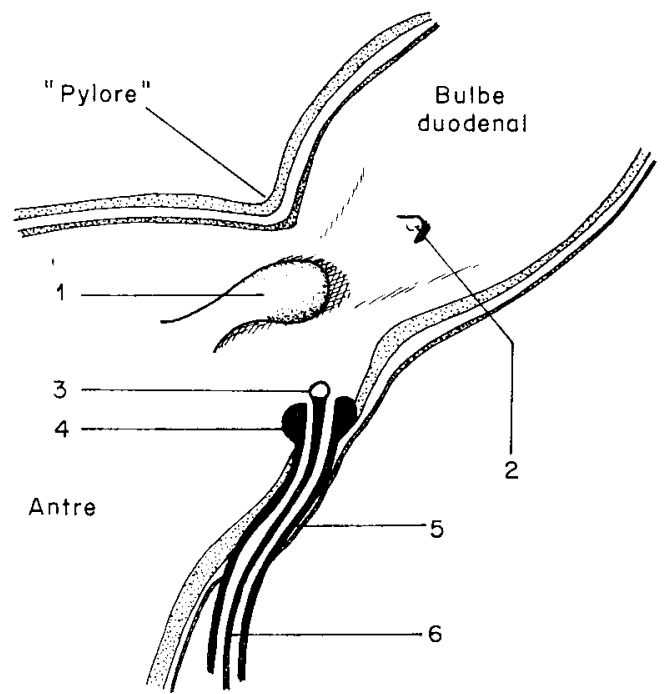

FIG. I. - Représentation schématique de la localisation juxta-pylorique de l'électrode stomacale I : Bouchon pylorique; 2 : Ampoule de Vater; 3 : Électrode de verre;

4 : Collerette distale du tube gastrique; 5 : Trajet sous muqueux du tube de silicone; 6: Câble de l'électrode

\section{TABLEAU I}

Composition centésimale des aliments standard

\begin{tabular}{|c|c|c|}
\hline $\begin{array}{l}\text { Matières premières } \\
\text { (p. 100) }\end{array}$ & $\begin{array}{c}\text { Croissance } \\
\mathrm{C}_{2}\end{array}$ & $\begin{array}{c}\text { Finition } \\
\mathrm{F}_{2}\end{array}$ \\
\hline Orge $\ldots \ldots \ldots \ldots \ldots \ldots$ & 35,00 & 66,50 \\
\hline Blé, ................. & 15,00 & - \\
\hline Maïs $\ldots \ldots \ldots \ldots \ldots \ldots$ & 15,00 & 一 \\
\hline Son $\ldots \ldots \ldots \ldots \ldots$ & 10,00 & - \\
\hline Remoulage gris $\ldots \ldots \ldots$ & $\ldots$ & 15,00 \\
\hline Tourteau de soja ....... & 18,00 & 12,00 \\
\hline Minéraux $\left({ }^{1}\right) \ldots \ldots \ldots \ldots$ & 2,90 & 2,50 \\
\hline Vitamines $\left({ }^{2}\right) \quad \ldots \ldots \ldots \ldots$ & 4,00 & 4,00 \\
\hline Antibiotiques.......... & 0,10 & - \\
\hline
\end{tabular}

(') Mélange minéral en p. 100 : Phosphate bicalcique 20,0 - Craie broyée 50,0 - Sel marin 20,0 - Carbonate de magnésium 7,73-Sulfate ferreux 1,00 - Sulfate de manganèse 0,60 - Sulfate de cuivre 0,15 - Sulfate de zine 0,50 - Sulfate de cobalt 0,01 - Iodure de potassium 0,01 .

( $\left.{ }^{2}\right)$ Mélange vitaminique (par $\mathrm{kg}$ de régime) : Vitamine A $4000 \mathrm{UI}$ Vitamine $D_{3} 1000$ UI - Riboflavine $2 \mathrm{mg}$ - Pantothénate de calcium $5 \mathrm{mg}$ -

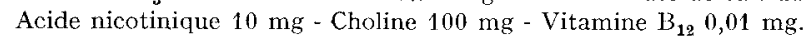


nage est effectué en fin d'enregistrement pour contrôler une éventuelle dérive de l'enregistreur.

Deux des aliments proposés sont identiques à ceux antérieurement utilisés (LAPLACE, I97I), soit un aliment dit de croissance $\left(\mathrm{C}_{2}\right)$ comportant une quantité appréciable de blé et de maïs et un aliment dit de finition $\left(\mathrm{F}_{2}\right)$ comportant une forte proportion d'orge à l'exclusion de toute autre céréale. Leurs compositions détaillées apparaissent dans le tableau $r$. Les aliments dits "lactosérum " sont de type finition et comportent $40 \mathrm{p}$. roo de lactosérum séché par le procédé Spray, soit doux (16 Dornic, 8,5 p. Ioo de matières minérales) soit naturellement acide $\left(57^{\circ}\right.$ Dornic, Io,5 p. Ioo de matières minérales). Leurs compositions détaillées apparaissent dans le tableau 2. Dans tous les cas, l'aliment est distribué sous forme de soupe (dilution du poids de farine dans 2 fois son poids d'eau). Le pH de la soupe proposée à l'animal est de 6,3 dans le cas de l'aliment $\mathrm{C}_{2}$, de 6,2 pour l'aliment $\mathrm{F}_{2}$, de 6,0 pour l'aliment lactosérum acide, et de 7,0 pour l'aliment lactosérum doux.

\section{TABLEAU 2}

Composition centésimale des aliments dits "lactosérum"

\begin{tabular}{|c|c|c|}
\hline \multirow{2}{*}{$\begin{array}{l}\text { Matières premières } \\
\text { (p. } 100)\end{array}$} & \multicolumn{2}{|c|}{ Lactosérum-finition } \\
\hline & Doux & Acide \\
\hline Blé . . . . . . . . . . & 2,00 & 2,00 \\
\hline Orge . . & 17,93 & 17,91 \\
\hline Soja 50 & 3,20 & 3,50 \\
\hline Son $\ldots \ldots \ldots \ldots \ldots$ & 35,00 & 35,00 \\
\hline Lactosérum . . . . . . & 10,00 & 40,00 \\
\hline Craie broyće ........ & 0,30 & - \\
\hline Oligo-éléments ${ }^{(1)} \ldots \ldots$ & 0,50 & 0,50 \\
\hline Vitamines $\left({ }^{2}\right) \quad \ldots \ldots \ldots$ & 1,00 & 1,00 \\
\hline Méthionine $\ldots \ldots \ldots$ & 0,07 & 0,09 \\
\hline
\end{tabular}

(1) Mélange d'oligo-éléments en p. 100 : Carbonate de magnésium 87,73Sulfate ferreux 8,00 - Sulfate de zinc $2,{ }^{\prime}+7$ - Oxyde de manganèse 0,52 Sulfate de cuivre 0,79 - Sulfate de cobalt 0,41 - Iodure de potassium stabilisé 0,08 .

(2) Mélange vitaminique en g par $k g$ : Pantothénate de calcium 2,5Riboflavine 1,0 - Acide ascorbique 1,0 - Nicotinamide 2,0 - Vitamine $B_{12}$ $(100 \mathrm{mg} / \mathrm{kg}) 20,0$ - Choline $(25 \mathrm{p} .100) 200,0$ - Vitamine A $(50000) 20,0$ Vitamine D (100 000) 2,5 - Blé (q.s.p. $1 \mathrm{~kg}$ ) 751,0.

Chaque animal a été soumis aux enregistrements pour 2 types d'aliments seulement. Les 2 aliments utilisés chez un même animal sont proposés en alternance au cours de 2 passages successifs, avec une période d'accoutumance de l'ordre de 4 jours entre chaque période expérimentale. La succession dans l'ordre chronologique des périodes expérimentales, définies par l'aliment utilisé, est donc décrite par l'un des 2 schémas suivants :

$\begin{array}{lccccc}\text { Aliments standard } & \ldots \ldots & \mathrm{F}_{2} & \mathrm{C}_{2} & \mathrm{~F}_{2} & \mathrm{C}_{2} \\ \text { Aliments lactosérum } \ldots \ldots & \text { Acide } & \text { Doux } & \text { Acide } & \text { Doux }\end{array}$

\section{RÉSULTATS}

\section{I. - Effets non spécifiques}

Au terme des $48 \mathrm{~h}$ de jeûne pré- et post-opératoire, soit $24 \mathrm{~h}$ après l'opération, le $\mathrm{pH}$ est en moyenne de 6,3 . Il est en moyenne de 5,4 le matin, $48 \mathrm{~h}$ après opération, chez l'animal réalimenté légèrement le jour précédent. Une période post-opératoire 
d'environ 4 jours est nécessaire pour que les porcs retrouvent à la fois une consommation quotidienne normale et le $\mathrm{pH}$ intragastrique voisin de 2 couramment observé le matin avant le premier repas.

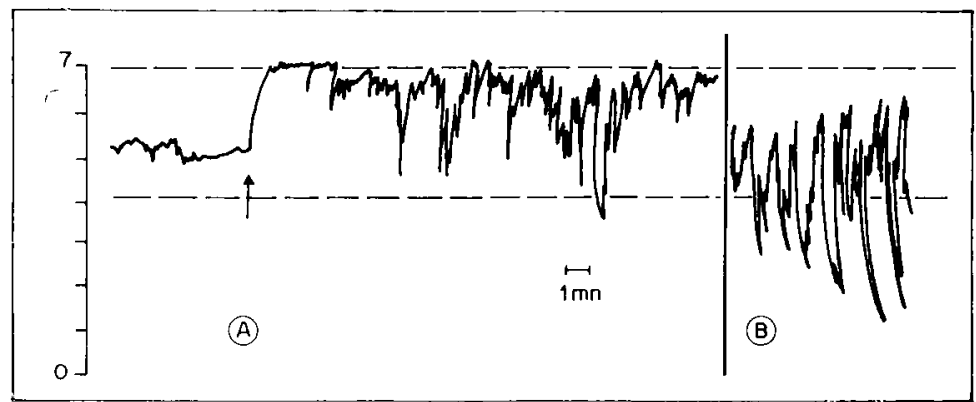

Fig. 2. - Influence de la localisation de l'électrode d'enregistrement. Située à $2,5 \mathrm{~cm}$ de l'extrémité distale du bouchon pylorique chez l'animal non alimenté depuis 10 heures l'électrode est avancée (en A) de $2 \mathrm{~cm}$ vers l'aval. En $B$, même localisation avancée chez l'animal alimenté

Les lignes pointillées indiquent l'étalonnage par des solutions tampons de pH 4,or et 6,86

Sur le plan technique, la localisation précise de l'électrode stomacale présente une grande importance à ce degré de proximité de la jonction gastroduodénale. Par rapport à la position habituelle d'enregistrement (la petite ampoule de verre de l'électrode d'enregistrement dépassant seule du tube de silicone) c'est-à-dire à $2,5 \mathrm{~cm}$ en amont de l'extrémité distale du bouchon pylorique, la propulsion de l'électrode de $2 \mathrm{~cm}$ plus en aval permet d'enregistrer une courbe totalement différente (fig. 2 ).

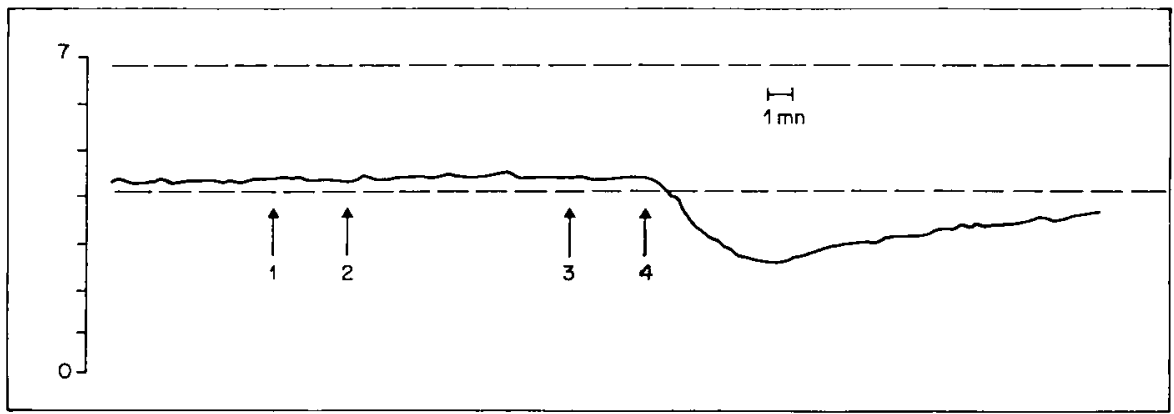

FIG. 3. - Distribution d'un aliment inhabituel et peu apprécié (lactosérum acide) chez un porc de $60 \mathrm{~kg}$ accoutumé à un aliment de finition standard

I, 2, 3 : Présentations successives avec refus du repas; en 4 : nouveau refus avec réaction aggressive de l'animal (solutions tampons de référence 4,0 I et 6,86 )

En effet, son niveau de base approche de la neutralité $(6,8$ à 7,0$)$. Ce tracé est hâché par de brutales déflections vers la zone d'acidité du milieu gastrique, correspondant au passage de jets de chyme évacué par l'estomac. Ces déflections, irrégulières en amplitude et en fréquence chez l'animal en période de repos digestif, apparaissent de façon extrêmement régulière à la suite d'un repas. Dans ce dernier cas, le $\mathrm{pH}$ n'atteint le plus fréquemment, dans l'intervalle des jets de chyme, qu'une zone de 5,8 à 6 . 
La période d'habituation à un nouvel aliment est généralement considérée comme nécessaire avant toute expérimentation. Mais l'intérêt d'une telle précaution est particulièrement évident si l'aliment est peu apprécié de l'animal. Le maintien de la présentation d'un aliment inhabituel suffit à produire (fig. 3) avec une réaction d'agressivité de l'animal, une hypersécrétion acide gastrique persistant 20 à $30 \mathrm{mn}$ malgré le retrait immédiat de l'aliment suscitant la réaction.

\section{2. - Aliment de finition-standard}

Lors de la distribution matinale $(9 \mathrm{~h})$, le $\mathrm{pH}$ intra gastrique est généralement compris entre $I, 5$ et 2,5 chez l'animal prématurément mis en alerte par les bruits du laboratoire (fig. 4). En l'absence d'une telle influence, on peut rencontrer un $\mathrm{pH}$ de l'ordre de 5 à 6 (le précédent repas ayant été distribué la veille à $\mathrm{I} 6 \mathrm{~h}$, soit $\mathrm{I} 7 \mathrm{~h}$ auparavant). Dans ce dernier cas (fig. 5) la préparation du repas suffit à provoquer une rapide diminution du $\mathrm{pH}$ qui rejoint la zone habituelle $(\mathrm{I}, 5$ à 2,5$)$. Par rapport à ce niveau de départ, le premier repas de la journée provoque toujours une remontée du $\mathrm{pH}$ d'une amplitude relativement constante, quelle que soit la quantité consommée, ou le $\mathrm{pH}$ préprandial. Cette amplitude est de 2,2 à 2,4 unités $\mathrm{pH}$ pour des repas de 600 à I $000 \mathrm{~g}$ de farine (additionnée de I 200 à $2000 \mathrm{~g}$ d'eau).

Par contre, le délai entre début du repas et début de la remontée $\mathrm{du} \mathrm{pH}$, ainsi que la rapidité de cette remontée sont essentiellement variables. On ne décèle pour ces caractéristiques aucune relation avec la rapidité d'ingestion donc la durée du repas, ni avec les facteurs individu ou niveau d'alimentation des jours précédents.

Lors du repas distribué à $\mathrm{I} 6 \mathrm{~h} 30$, la variation de $\mathrm{pH}$ observée est toujours très amortie. La remontée est progressive et d'une amplitude beaucoup plus limitée puisque le $\mathrm{pH}$ évolue le plus souvent de 2,5 à 3,5 . La courbe obtenue dans l'intervalle des 2 repas de la journée traduit une décroissance régulière du $\mathrm{pH}$ de 3,8-4,8 (après repas du matin) à environ 2,5 en l'espace de 2 heures, sans accident notable du tracé. I,e $\mathrm{pH}$ reste alors stationnaire jusqu'au second repas.

Le fractionnement de la ration quotidienne en 3 voire 4 repas ne modifie pas l'aspect caractéristique du premier repas d'une part, du ou des autres repas d'autre part.

\section{3. - Aliment de croissance standard}

Là encore, la distribution du repas du matin produit un effet important. Le $\mathrm{pH}$ initial compris entre $I, O$ et 3,0 , mais le plus souvent de 1'ordre de $I, 0$ à $I, 5$, présente une remontée précoce. Celle-ci débute en effet toujours durant la première moitié de la durée du repas. Cette variation est par ailleurs plus rapide que pour l'aliment finition, et peut s'effectuer en l'espace de 2 minutes. Elle est enfin d'une amplitude plus grande, de l'ordre de 3 unités $\mathrm{pH}$ pour des repas représentant 600 à I ooo g d'aliment sec. Cette amplitude peut occasionnellement atteindre 4 unités $\mathrm{pH}$ pour des repas nettement plus importants (I $800 \mathrm{~g}$ d'aliment sec).

Au-delà de cette variation initiale, l'évolution du $\mathrm{pH}$ au cours de la période de $9 \mathrm{~h}$ à $\mathrm{I} 7 \mathrm{~h}$ est très lente (fig. 6). Restée en plateau à la valeur atteinte après le repas, la courbe présente après $I$ à $2 \mathrm{~h}$ une décroissance progressive jusqu'à un minimum qui est atteint 2 à $4 \mathrm{~h}$ après le repas (réduction moyenne de 2 à 2,5 unités $\mathrm{pH}$ ). L,e $\mathrm{pH}$ 


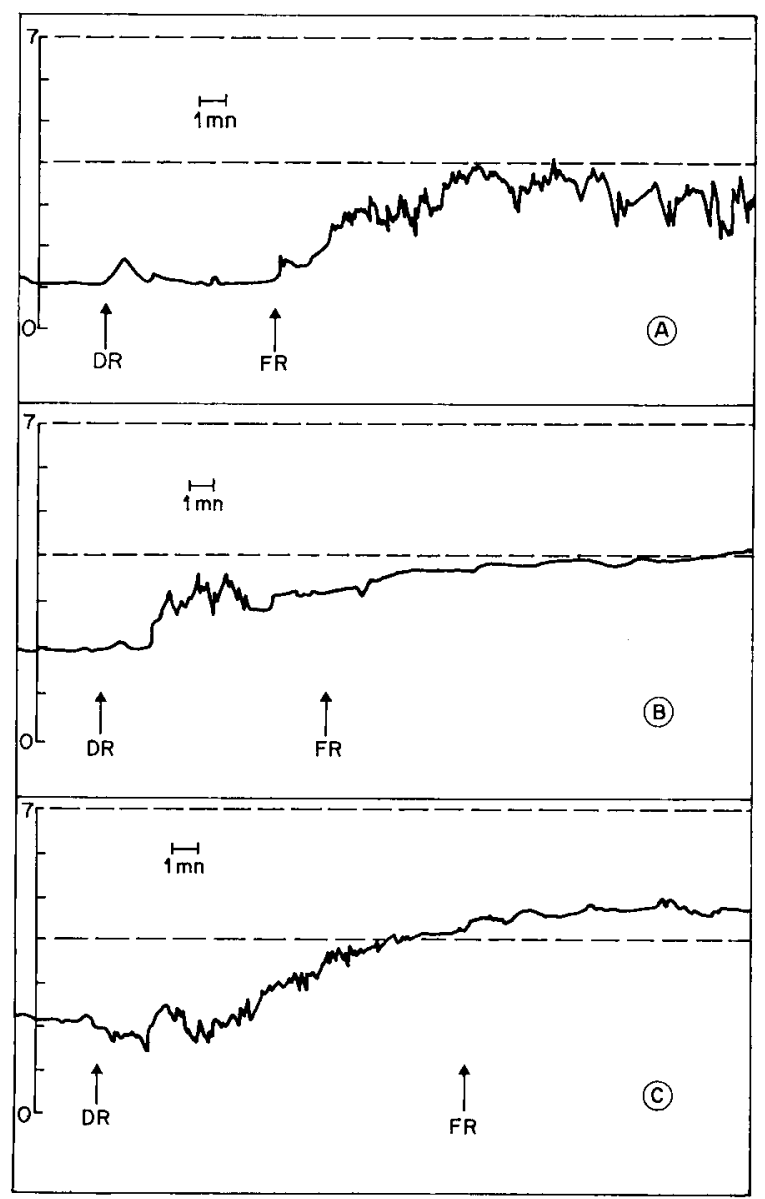

FIG. 4. - Effet de la distribution à $9 \mathrm{~h}$ d'un repas d'un $\mathrm{kg}$ d'aliment finition standard (dilué par 2 fois son poids d'eau) chez un même animal, 3 jours différents :

A : délai long : amplitude de la variation $\mathrm{pH} \quad \mathrm{I}, 5$ à 3,8 , évolution progressive ;

$\mathrm{B}$ : délai bref : amplitude pH $\mathrm{I}, 8$ à 4,0 , évolution très lente;

$\mathrm{C}$ : délai bref : amplitude $\mathrm{pH} 2,3$ à 4,7 , évolution progressive.

On entend ici par " délai "le temps écoulé entre le début du repas et le début de l'alcalinisation, et par "évolution" la rapidité plus ou moins grande avec laquelle la courbe atteint, à partir du début de l'alcalinisation, le maximum de celle-ci.

DR: Début du repas;

FR : Fin du repas (solutions tampons de référence 4,or et 7,00 )

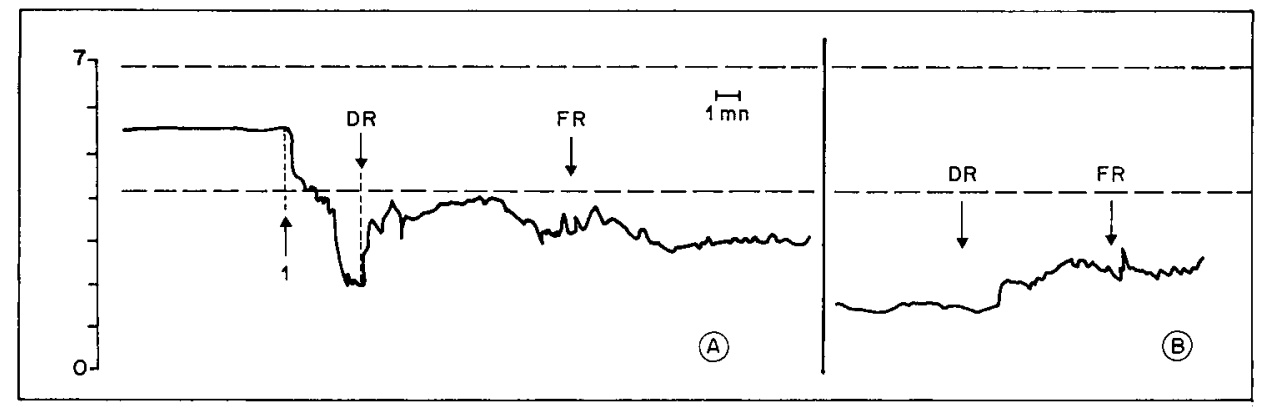

FIG. 5. - Effets de repas de $600 \mathrm{~g}$ d'aliment finition standard (dilué par 2 fois son poids d'eau). En $A$, on note l'effet de la préparation de la ration ( $\mathrm{I}$ ) et celui du repas matinal ( $9 \mathrm{~h}$ ). En $B$, on observe l'effet d'un même volume de repas distribué à $16 \quad h 30$

DR : Début du repas;

FR: Fin du repas (solutions tampons de référence 4 ,or et 6,86 ). 


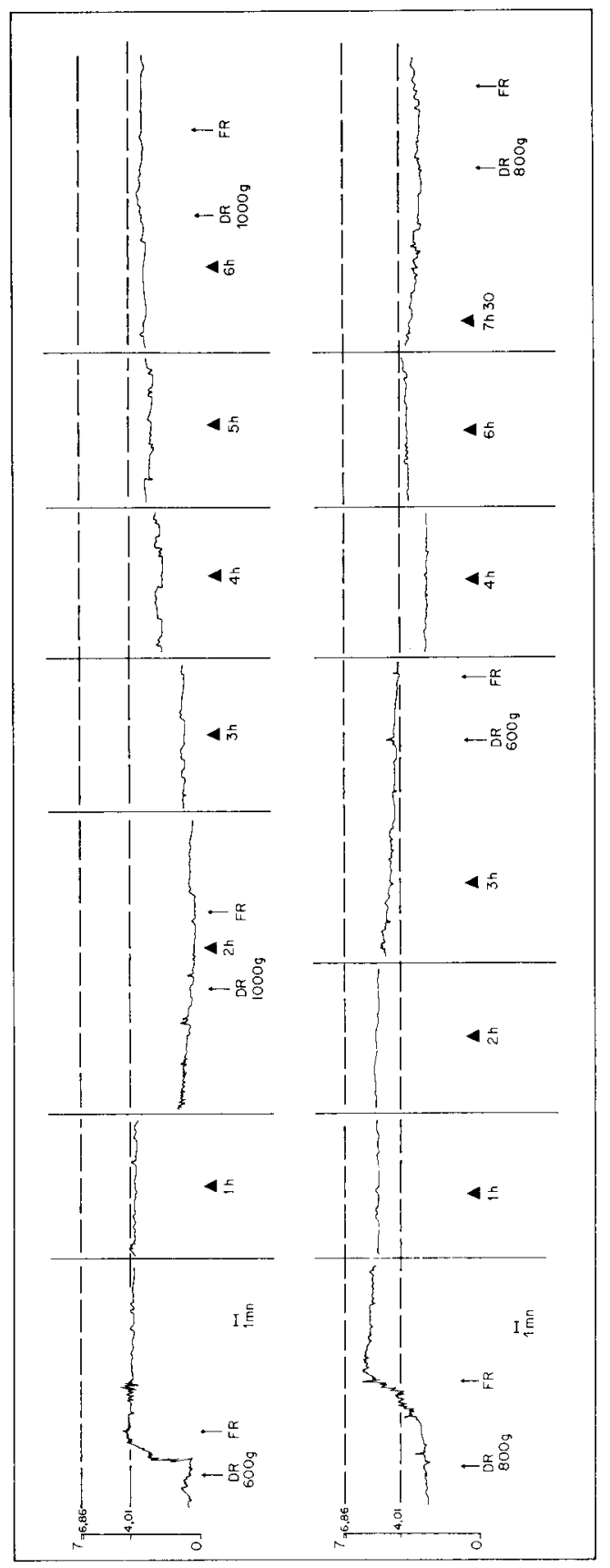

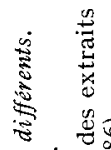

औ $\Xi 0^{\circ}$

융효

क्ड है:

ङ

चै के

害言 总总嵒

造, 步

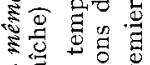

密要递

ช⿺辶大

密产要寻

. ज्ञ

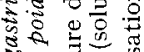

S:

놀 농

Aี

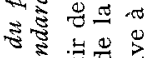

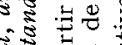

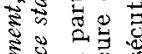

농 $\pi$

空

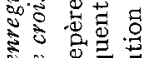

के

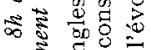

\&

ह 520

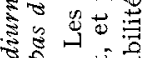

के $\dot{1} \dot{0}$

क्ष

$\circ$ o

A

造

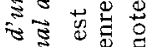

곤

o 8 in $=0$

है \&ै

ثิ

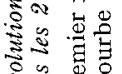

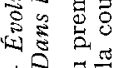

$18 \exists$

$\circ$.

○े 
présente ensuite une lente remontée de $\mathrm{I}, \mathrm{O}$ à $\mathrm{I}, 5$ unités $\mathrm{pH}$, ramenant le $\mathrm{pH}$ enregistré à $\mathrm{I} 7 \mathrm{~h}$ à une valeur comprise entre $\mathrm{pH} 3$ et $\mathrm{pH}_{4}$.

Cette évolution générale n'est pas modifiée par les autres repas de la journée, quels qu'en soient le nombre et l'importance volumétrique. En effet, à la différence de l'aliment finition, les repas autres que le repas du matin sont pratiquement dépourvus de tout effet visible sur le $\mathrm{pH}$ du contenu gastrique pré-pylorique.

\section{4. - Nombre, intervalle et volume des repas}

Cette apparente absence de modifications nous a incité à multiplier les combinaisons différentes dans le cas de l'aliment de croissance, quoique le nombre des repas n'ait jamais influencé les résultats obtenus avec l'un ou l'autre des aliments. En pratique, un tel problème peut être subdivisé pour l'expérimentation en 3 facteurs étroitement interdépendants, illustrés par la figure 7 : intervalle entre les repas, volume du repas d'épreuve, volume du repas précédent déterminant le volume gastrique résiduel.

La distribution d'un second repas de mêmes caractéristiques à des intervalles variables après le précédent repas, ne provoque pas de variation nette reproductible, bien que les volumes gastriques résiduels soient certainement très différents. Si l'on agit sur ce volume gastrique résiduel en utilisant des repas de volumes variant dans le rapport de I à 2,5 , antérieurement à un même repas d'épreuve distribué au terme d'un délai constant, on constate que les courbes de $\mathrm{pH}$ ne sont pas sensiblement différentes.

Enfin, pour un même horaire de distribution, la modification dans un rapport de I à 3 de la quantité ingérée au premier repas n'affecte la courbe de $\mathrm{pH}$, dans la majorité des cas, que de façon très limitée. Une remontée du pH d'une amplitude supérieure à 3 unités se présente en fait, même pour un repas de I $800 \mathrm{~g}$, comme une variation exceptionnelle. De la même façon, on peut aussi considérer comme très exceptionnelle, même pour un repas de $600 \mathrm{~g}$, une remontée n'excédant pas 2,5 unités $\mathrm{pH}$.

\section{5. - Les aliments lactosérum-finition}

Une caractéristique commune aux deux types d'aliments contenant du lactosérum est que le premier repas de la journée induit une augmentation importante du $\mathrm{pH}$ intra gastrique (fig. 8), mais aussi que les autres repas produisent toujours une certaine augmentation (fig. 9). En cela, ces aliments de finition à 40 p. Ioo de lactosérum sec se comportent donc comme l'aliment de finition standard.

Une seconde caractéristique commune aux aliments incorporant du lactosérum réside dans le fait que la variation ascendante $\mathrm{du} \mathrm{pH}$, à l'occasion de tous les repas quel qu'en soit l'horaire, débute toujours dans les 2 à 3 premières minutes de l'ingestion alimentaire, particularité qui rapproche ces effets de ceux de l'aliment de croissance standard. Quel que soit 1'aliment utilisé, le $\mathrm{pH}$ intragastrique chez l'animal encore à jeûn le matin est toujours situé dans la même zone entre $\mathrm{pH}$ r,5 et $\mathrm{pH} 3,0$.

Certaines différences constantes permettent cependant d'identifier les 2 types d'aliments lactosérum. La forme de la courbe ascendante de $\mathrm{pH}$ lors du repas du matin (fig. 8), dans le cas d'incorporation de lactosérum acide, rappelle en tous points 


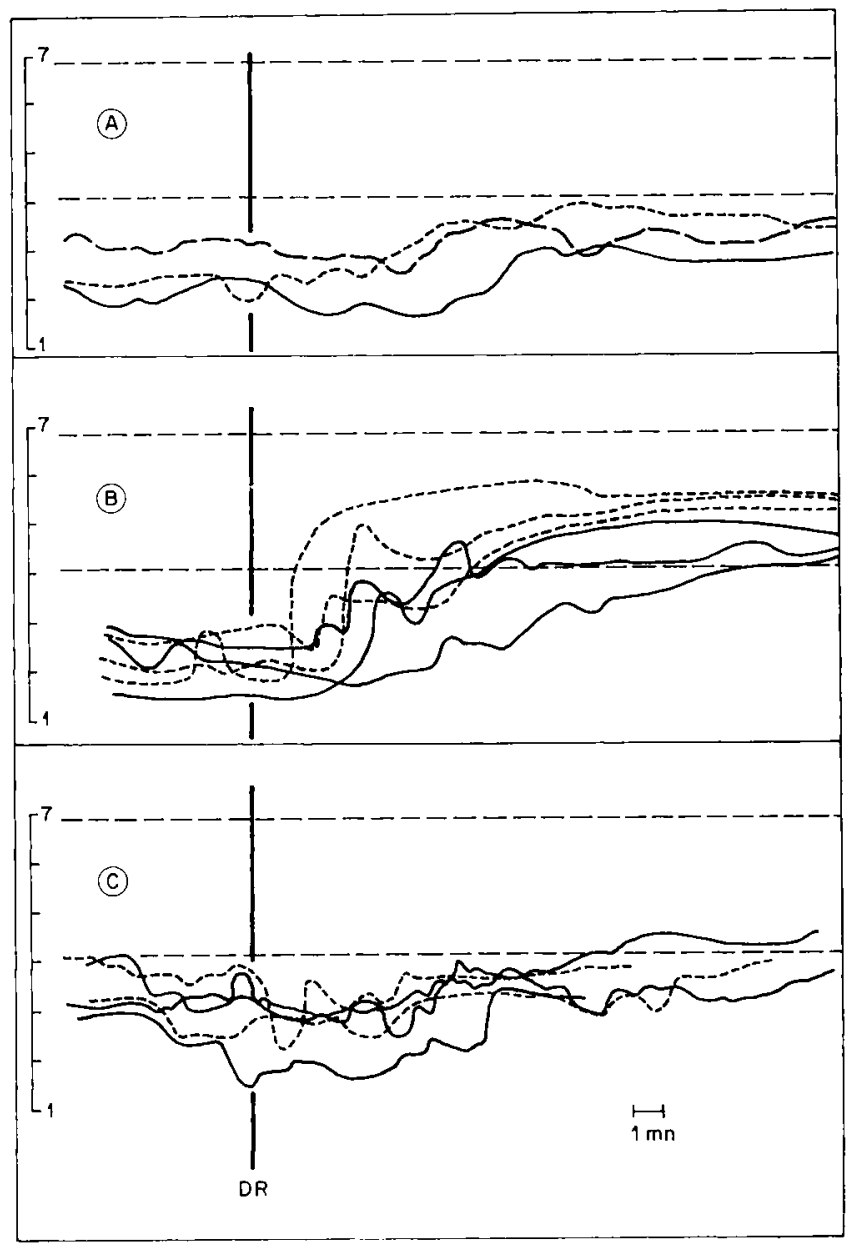

FiG. 7

A : Effet de l'intervalle entre les repas : distribution d'un repas de $600 \mathrm{~g}$ d'aliment sec de croissance, selon les cas I $\mathrm{h}\left(-\frac{}{-}\right), 2 \mathrm{~h}(----)$ ) ou $3 \mathrm{~h}(---)$ après un premier repas identique (6oo g d'aliment croissance à $9 \mathrm{~h}$ ).

B : Effet du volume du repas : courbes de $\mathrm{pH}$ obtenues dans tous les cas pour le repas d'aliment croissance à $9 \mathrm{~h}$. La quantité consommée est selon les cas de $600 \mathrm{~g} \mathrm{(-—)} \mathrm{ou} \mathrm{de} \mathrm{I} 800 \mathrm{~g} \mathrm{(-- \ldots )}$ d'aliment sec.

$\mathrm{C}:$ Effet du volume gastrique résiduel : courbes de $\mathrm{pH}$ obtenues dans tous les cas pour un repas à I $2 \mathrm{~h}$, de $600 \mathrm{~g}$ d'aliment croissance. La quantité consommée lors du précédent repas (9 h) a été selon les cas de $600 \mathrm{~g}(-(-)$ ou de I $500 \mathrm{~g}(-\ldots)$. (Solutions tampons de référence 4 , or et 6,86$)$. 


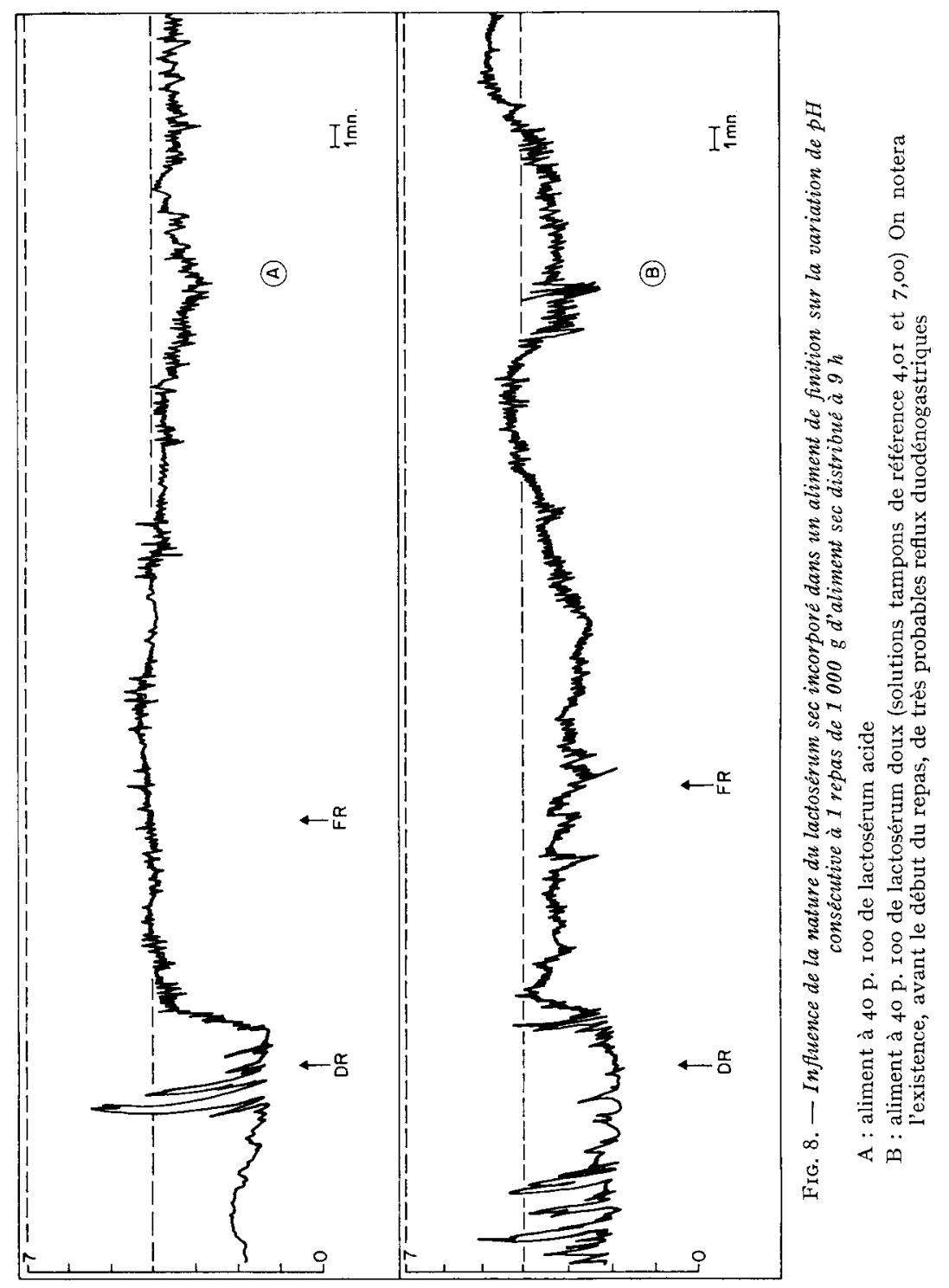


celle observée avec l'aliment standard de croissance. Elle est en effet extrêmement rapide voire brutale, et d'une amplitude similaire de l'ordre de 3 unités $\mathrm{pH}$. Lors d'incorporation de lactosérum doux, cette même remontée du $\mathrm{pH}$ lors du r er repas ne développe jamais d'emblée une pleine amplitude. Celle-ci n'est atteinte qu'en 2 ou 3 vagues successives, et au bout de 30 à $70 \mathrm{mn}$, alors que le maximum est pratiquement toujours atteint dès la fin du repas lactosérum acide. Si l'on fait abstraction de cette différence caractéristique de décours du $\mathrm{pH}$ dans 1'heure qui suit le premier repas, on peut reconnaitre aux deux variations une amplitude semblable d'environ 3 unités $\mathrm{pH}$.

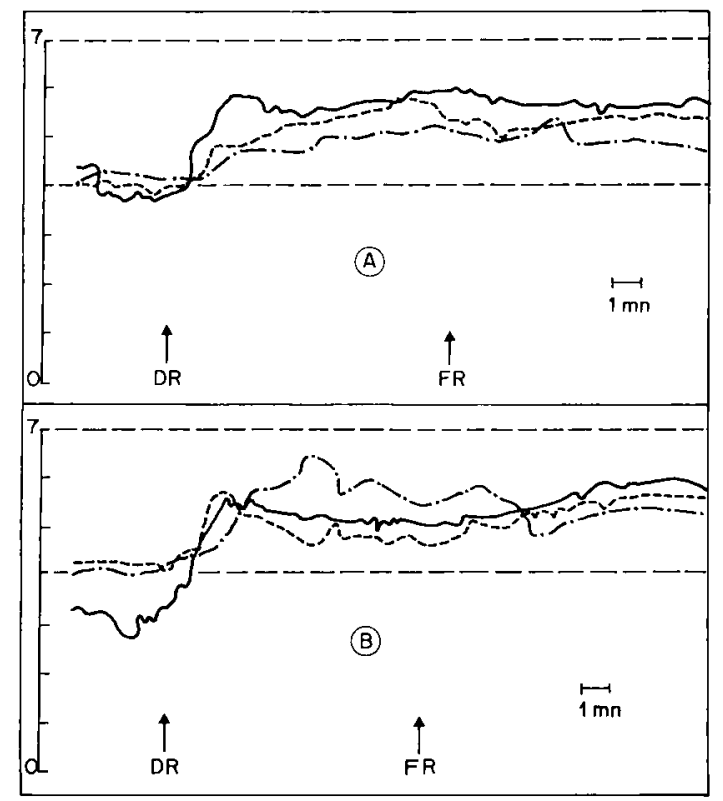

FIG. 9. - - Effets respectifs de 3 repas de $1000 \mathrm{~g}$ d'aliment sec distribués dans une même journée sur la courbe de $p H$ intragastrique, en $A$ dans le cas d'un aliment à $40 p$. 100 de lactosérum acide, en $B$ dans le cas d'un aliment à 40 p. 100 de lactosérum doux.

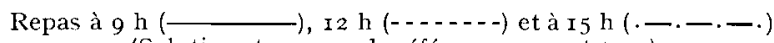

(Solutions tampons de référence 4,0 I et 7,00 )

Pour faciliter la comparaison de l'amplitude de la variation induite par chaque repas ( $9 \mathrm{~h}, \mathrm{I} 2 \mathrm{~h}, \mathrm{I} 5 \mathrm{~h}$ ), l'enregistrement à $9 \mathrm{~h}$ a été choisi avec un $\mathrm{pH}$ initial aussi proche que possible des pH observés avant les repas de $\mathrm{I} 2 \mathrm{~h}$ et $\mathrm{I} 5 \mathrm{~h}$.

En ce qui concerne les variations induites par le ou les autres repas distribués dans la journée, elles sont souvent, quel que soit le lactosérum utilisé, d'amplitude supérieure à ce qui est observé pour des repas d'aliment finition standard distribués dans des conditions identiques. L'amplitude de ces variations est en effet le plus souvent de $\mathrm{I}, 5$ à 2 unités $\mathrm{pH}$ (fig. 9). D'une façon générale, ces variations sont d'autant moins accusées que le repas survient en un temps où le $\mathrm{pH}$ se trouve dans une zone plus haute de la courbe enregistrée dans la journée. L'aspect général de cette courbe entre $9 \mathrm{~h}$ et $\mathrm{I} 7 \mathrm{~h}$ se présente comme un tracé ondoyant, chaque repas entraînant un rehaussement du $\mathrm{pH}$ plus ou moins important, et qui conduit à une valeur voisine de $\mathrm{pH}_{4}$ à $\mathrm{I}_{7} \mathrm{~h}$ pour des repas distribués à 9 , I 2 et $\mathrm{I} 5$ heures. 
Une dernière particularité mérite d'être mentionnée. Lors de distribution d'aliments comportant du lactosérum et tout particulièrement du lactosérum doux, on constate l'apparition par salves d'importantes déflections de la courbe de $\mathrm{pH}$ vers la neutralité, susceptibles de représenter des reflux duodéno-gastriques. Ces phénomènes apparaissent presque toujours avant le repas du matin (fig. 8) et réapparaissent périodiquement dans la journée au-delà de 90 à $\mathrm{I} 20 \mathrm{mn}$ après le premier repas. Un épisode fréquemment important se situe alentour de $\mathrm{I}_{4} \mathrm{~h}$. La multiplication des repas dans la journée tend à effacer ces accidents du tracé puisqu'ils disparaissent durant $90 \mathrm{mn}$ environ après chaque repas.

Précisons enfin qu'aucune différence n'a été décelée dans les effets d'un même aliment entre les passages successifs dans le cadre de l'alternance expérimentale.

\section{DISCUSSION}

Sur le plan méthodologique, plusieurs points particuliers doivent être soulignés. En ce qui concerne le site d'enregistrement, le choix de la région antrale pré-pylorique est guidé par l'intérêt porté à l'influence du chyme évacué par l'estomac dans le contrôle de cette évacuation. Cette localisation implique que nos résultats ne peuvent être superposés de façon directe à ceux de LAWRENCE (I97o) même pour un régime analogue, essentiellement à base d'orge, puisque le $\mathrm{pH}$ est susceptible de présenter des variations notables selon le site d'enregistrement au niveau de 1'estomac (RoveLSTAD, OWEN et MAGATH, I952). Vis-à-vis de la technique chirurgicale mise en œuvre, l'anatomie et l'importance fonctionnelle de la région antrale justifient également qu'ait été retenu le principe d'un simple tube gastrique permettant de faire cheminer directement l'électrode stomacale. Assurément, l'usage d'une classique canule gastrique, telle que celles mises en place par LAWRENCE (I970) au niveau de la jonction fundus-antre, aurait permis de réunir les électrodes stomacale et de référence et d'éviter une fistulation cæcale additionnelle. Mais cette dernière est toujours parfaitement tolérée pendant des périodes très prolongées, et l'électrode de référence utilisée est habituellement, chez l'Homme, mise en place dans un orifice naturel (boucheanus). Aussi, 1'usage du tube gastrique nous parait-il préférable à une canule susceptible de gêner assez considérablement 1'activité motrice antrale. Certes, toute fistulation gastrique peut être évitée par 1'usage de la technique de l'endoradiosonde déglutie, permettant des enregistrements continus de grande qualité illustrés chez le Chien par le travail de LABRID et al. (I973). Mais la simple limitation du déplacement de l'endoradiosonde par un fil de nylon interdisant le franchissement du pylore n'assure pas la localisation intragastrique recherchée dans notre cas. L'usage du tube gastrique offre donc l'avantage d'une localisation précise permanente de l'électrode stomacale qui coiffe l'orifice du tube. Ia collerette d'élastomère cerclant cette extrémité du tube assure par ailleurs le maintien d'un écartement constant de la surface muqueuse, évitant tout contact indésirable de l'électrode et de la muqueuse. Il n'est ainsi pas nécessaire d'utiliser un système de protection du bulbe de verre de l'électrode stomacale tel que celui utilisé par RUNE (I968). On peut du reste noter l'identité des tracés recueillis par cet auteur chez 1'Homme au niveau de l'antre et du pylore 
et de ceux que nous avons enregistrés chez le Porc en des sites homologues. Pour en terminer avec la méthodologie, on peut remarquer que le relevé du $\mathrm{pH}$ toutes les 5 minutes (LAWRENCE, I97O) est susceptible d'éliminer certaines variations brèves de peu d'intérêt vis-à-vis du décours du $\mathrm{pH}$ au fil d'une journée, mais risque également de laisser échapper des phénomènes d'un intérêt certain, tels que les reflux duodéno-gastriques. De ce fait, l'enregistrement continu d'un signal filtré et débarassé des seules variations très brèves telles que celles liées au passage des ondes contractiles, représente la solution la plus satisfaisante.

Si l'on examine très globalement l'aspect des courbes obtenues au cours de la période diurne $9 \mathrm{~h}-\mathbf{I} 7 \mathrm{~h}$, on peut constater qu'à partir d'une zone de $\mathrm{pH}$ très acide, le premier repas exerce un effet d'induction majeur de la principale variation observée. Le relèvement ainsi provoqué de la courbe de $\mathrm{pH}$ est ensuite plus ou moins soutenu (mais très exceptionnellement seulement renforcé) selon le nombre des repas et la nature de l'aliment proposé. La question reste posée de savoir si la courbe de $\mathrm{pH}$ évolue de façon régulière entre l'acidité moyenne observée à $\mathrm{I} 7 \mathrm{~h}$ et la forte acidité généralement observée à $9 \mathrm{~h}$, ou si elle présente d'autres accidents notables. Ceci revient à se demander si le premier repas est véritablement l'inducteur d'une variation cyclique du $\mathrm{pH}$, ou s'il n'intervient que pour synchroniser un rythme autre dans lequel l'alternance veille-sommeil jouerait un rôle non négligeable (BAUst et RoHrwasser, I969). Sur ce point, la comparaison des courbes de $\mathrm{pH}$ intra gastrique au cours du nycthémère entre des porcs en alimentation ad libitum vraie et des porcs soumis à un rythme de repas diurnes imposés, présentera le plus grand intérêt. L'éventualité d'une pathologie gastrique induite par certains modes d'alimentation n'est pas à exclure, indépendamment d'aspects très classiques liés à 1'aliment (finesse de mouture, taux de cellulose).

L'existence occasionnelle de soudaines déflections du $\mathrm{pH}$ intra-gastrique acide vers la neutralité semble bien devoir être rapportée à l'existence de reflux duodénaux. A défaut de démonstration, certaines présomptions inclinent dans ce sens : évolution symétrique du $\mathrm{pH}$ duodénal voisin de la neutralité vers une acidification lors des émissions de chyme gastrique ; courbe crénelée avec fortes oscillations du pH intragastrique chez les chiens " hyperchlorhydriques" (LABRID et al., I973). Si l'on admet une telle interprétation, il faut souligner que la présence de salves de reflux duodénogastriques, n'a été observée que pour les aliments comportant du lactosérum, ce qui constitue une particularité dont la cause reste totalement obscure. Tout au plus peut on remarquer l'amplitude de ces ondes. Si l'on en juge par les courbes de $\mathrm{pH}$ observées dans le bulbe duodénal ou le duodénum, chez l'Homme ou le Chien, par Rune (rg68) et par Brooks et Grossman (I970), il paraît probable que ces reflux proviennent d'un territoire nettement plus distant du pylore que le bulbe duodénal. Cependant, chez le Porc, le canal cholédoque débouche particulièrement près du pylore, et ces ondes de $\mathrm{pH}$ pourraient correspondre à une remontée de bile. On note dans ce sens que la présence de quantités relativement importantes de bile est fréquemment observée dans l'estomac de porcs à jeûn. Mais les inconnues relativement au $\mathrm{pH}$ du milieu duodénal restent trop nombreuses (RUNE, I973) pour permettre une interprétation immédiate.

L'étude plus particulière de l'effet du repas dans l'heure qui suit le début de celui-ci permet de rassembler un certain nombre d'aspects caractéristiques de l'aliment utilisé. Cette observation confirme semblable affirmation de LAWRENCE (I970) relativement aux effets nettement distincts de régimes de technologie différente ou à 
l'obtention de $\mathrm{pH}$ plus faibles avec les aliments à base d'orge ou présentés sous forme humide, par rapport à des aliments à base de maïs ou présentés sous forme sèche (LAWRENCE, I972). Il apparait cependant que cette affirmation doit être nuancée puisque si l'aliment croissance (orge, maïs, blé) induit bien la variation la plus importante et la plus brutale lors du $\mathrm{I}^{\mathrm{er}}$ repas, il n'en induit aucune pour les autres repas de la journée. Par contre, l'aliment de finition (orge) commeles aliments de finition comportant du lactosérum induisent tous une variation pour chacun des repas. Les causes des particularités qui identifient les effets de chacun des aliments restent totalement obscures. Parmi les facteurs possibles on peut envisager le pouvoir tampon de ces aliments, l'importance des sécrétions salivaire et gastrique selon 1'appétibilité particulière à chaque ration; le rythme d'évacuation gastrique peut enfin constituer un facteur déterminant de la décroissance progressive du $\mathrm{pH}$ à la suite de l'augmentation initiale au moment du repas. Il devient alors difficile de déterminer dans quelle mesure le $\mathrm{pH}$ peut lui-même participer au contrôle de l'évacuation gastroduodénale.

\title{
CONCLUSIONS
}

L'évolution du pH intra gastrique pré-pylorique chez le Porc en croissance-finition est principalement marquée par l'effet du $\mathrm{I}^{\mathrm{er}}$ repas de la journée. Le nombre des repas, leur importance quantitative, leur vitesse d'ingestion, n'ont pas d'effet déterminant. Par contre, la composition de l'aliment constitue une source de variations qui fournit aux courbes de $\mathrm{pH}$ une configuration caractéristique de chacune des formulations utilisées. Les informations descriptives apportées par la technique mise en œuvre peuvent être précieuses dans l'analyse de phénomènes pathologiques d'origine alimentaire, mais ne permettent guère d'en tirer des conclusions quant au processus d'évacuation gastrique.

Reçu pour publication en août 1973.

\section{SUMMARY}

\author{
REGISTRATION OF INTRAGASTRIC PH AND ITS VARIATIONS ACCORDING \\ TO THE, TYPE, VOI,UME AND FREQUENCY OF MEALS IN FINISHING PIGS
}

In order to study the importance of $\mathrm{pH}$ as factor controlling the gastric emptying in pig, a comparative in vivo registration of intragastric $\mathrm{pH}$ was made on 6 Lavge White pigs weighing 60 to $68 \mathrm{~kg}$ live weight. These pigs were fitted with a permanent gastric tubing and a permanent cacal cannula. The glass-electrode, specifically designed for measuring $\mathrm{pH}$ changes in the gastroduodenal area, was introduced through gastric tubing and localized at $2 \mathrm{~cm}$ above the pyloric ring. The reference-electrode was kept in the cæcal fistula. In vivo gastric pH changes were registered through a $\mathrm{pH}$ meter on a polygraph giving an uninterrupted curve.

Four diets were used : a standard diet for growing pigs (35 p. Ioo barley, I5 p. Ioo corn, I5 p. Ioo maize) ; a standard diet for finishing pigs (66.5 p. Ioo barley) ; and two diets for finishing pigs incorporating $40 \mathrm{p}$. Ioo of dried whey, soft ( $16^{\circ}$ Dornic acidity) or acid $\left(57^{\circ}\right.$ Dornic acidity). The pigs, housed in a metabolism cage, were fed on a semi liquid form (weight dilution : flour I, water 2). 
The $\mathrm{pH}$ before the first meal (9 a.m.) was about a value of 2.00 ( 1.0 to 3.0 ). At this first morning meal, the standard diet for finishing pigs gave an increment of 2,2 to $2.4 \mathrm{pH}$ units. Thus the increase began after a variable post prandial delay, and was more or less fast. In the case of the standard diet for growing pigs, such an increase was observed, but it was ever very fast, earlier and of greater amplitude $(2.5$ to $3 \mathrm{pH}$ units). On the other hand, the other meals throughout the day gave only a small increase (standard-finishing pigs) or no effects (standardgrowing pigs).

In the case of the diets incorporating whey, the increase at the first meal was ever of about $3 \mathrm{pH}$ units; the other meals gave, whatever the whey, an increase of $\mathbf{I} .5$ to $2 \mathrm{pH}$ units; The most obvious characteristic was that, at the first meal, the increase was fast and early in the case of the acid whey, and slow (30 to $70 \mathrm{mn}$ ) in the case of the soft whey.

Whatever may be the diet used, neither the number of meals, nor the volume of the meal gave a significant effect on the $\mathrm{pH}$ curve.

It was concluded that each diet gave a distinct pattern. But such a study brought no sufficient information on the $\mathrm{pH}$ as factor controlling the gastric emptying.

\section{RÉFÉRENCES BIBLIOGRAPHIQUES}

Baust W., Rohrwasser W., 1969. Das Verhalten von pH und Motilität des Magens im natürlichen Schlaf des Menschen. Pfügers Arch., 305, 229-240.

Brooks A. M., Grossman P. I., I97o. Postprandial pH and neutralizing capacity of the proximal duodenum in dogs. Gastroenterology, 59, 85-89.

Derrien A., Gallant D., Aumaitre A., r97i. Dégradation in vitro de différents amidons (mais, manioc, pomme de terre) par le suc pancréatique du porcelet sevré à 5 semaines. Étude microscopique. Ann. Biol. anim. Bioch. Biophys., 11, 326-330.

Février C., Rérat A., Aumaitre A., rg7r. Étude in vivo de l'absorption des glucides à l'aide d'une méthode en continu chez le Porc. Ann. Biol. anim. Bioch. Biophys., 11, 352-353.

Labrid D., Duchêne-Marullaz P., Dureng G., Baissat J., I973. Étude du pH gastrique du chien Beagle à jeûn et de ses variations après absorption de bicarbonate de sodium. J. Physiol., Paris, 66, $3 I-42$.

Laplace J. P., I97I. Premières observations sur la motricité gastroduodénale en tant que facteurs de contrôle de l'évacuation gastrique chez le Porc. Xe Congrès Int. Zootech., Versailles.

Laplace J. P., I972. Motricité gastro intestinale chez le Porc : Étude descriptive par électromyographie et corrélations nutritionnelles. Rec. Med. vet., 148, 37-6r.

Laplace J. P., Tomassone R., r97o. Évacuation gastro duodénale chez le Porc. Fistulation chronique par voie thoracique extrapleurale, recherche d'une technique d'analyse mathématique de l'évacuation. Ann. Zootech., 19, 303-332.

LAWRENCE T. L. J., I97o. Some effects of including differently processed barley in the diet of the growing pig. II. In vivo gastric $\mathrm{pH}$ changes. Anim. Prod., 12, I5 I-I63.

LAWRENCE T, L. J., I972. The effect of certain dietary factors on in vivo $\mathrm{pH}$ changes and pepsin activity in the stomach of the growing pig. Br.Vet.J., 128, 402-41 r.

Markowitz J, Archibald J., Downie H. G,. 1964. Experimental surgery. Vifi. Gastric fistulae, I2I-142, I vol., Williams and Wilkins Co. Ed., Baltimore.

Rérat A., Aumaitre A., ig7r. Mesure de l'absorption des glucides chez le Porc en croissance. $X^{e}$ Congrès Int. Zootech. VII. Nutrition Porcs, Versailles.

Rovelstad R. A., Owen C. A., Magath T. B., 1952. Factors influencing the continuous recording of in situ pH of gastric and duodenal contents. Gastroenterology, 20, 609-624.

Rune S. J, I968. An electode for $\mathrm{pH}$ measurement in the gastrointestinal tract. Scand. J. Gastroent., 3, 9 r -94 .

RunE S. J., 1973. $\mathrm{pH}$ in the human duodenum. Its physiological and pathophysiological significance. Digestion, 8, 26I-268. 